\title{
Fast Cognitive Decline at the Time of Dementia Diagnosis: A Major Prognostic Factor for Survival in the Community
}

\author{
Laure Carcaillon $^{a, b}$ Karine Pérès ${ }^{a, b}$ Jean-Jacques Péréd Catherine Helmer ${ }^{a, b}$ \\ Jean-Marc Orgogozo ${ }^{a-c}$ Jean-François Dartigues ${ }^{a-c}$ \\ ${ }^{\mathrm{a}}$ Inserm, Unit 593, ${ }^{\mathrm{b}}$ University Victor Segalen and ${ }^{\mathrm{C}}$ University Hospital Center, Bordeaux, and \\ ${ }^{\mathrm{d}}$ Novartis Pharma SAS, Rueil-Malmaison, France
}

\section{Key Words}

Fast cognitive decline $\cdot$ Mortality rate $\cdot$ Alzheimer's disease

\begin{abstract}
Background/Aims: Current findings suggest the existence of a category of fast cognitive decliners with a poorer prognosis but better treatment response. Our study aimed at confirming the concept of fast decliners at the time of Alzheimer's disease (AD) diagnosis which best predicts mortality, in an unselected sample. Methods: 245 incident cases of $A D$ were selected from the French longitudinal cohort PAQUID. We investigated a different threshold of cognitive decline [measured by the annual loss of points in the Mini Mental State Examination (MMSE) score] to define when a subject could be considered as a fast decliner. We used Cox proportional hazards models to study the relation between cognitive decline and mortality. Results: The significant threshold of decline associated with a higher mortality rate was a loss of 3 points per year in the MMSE score. Among the 245 AD cases, 83 (33.9\%) subjects were considered as fast decliners. Of them, $78.3 \%$ died during the follow-up compared with $63.0 \%$ of the slow decliners ( $\mathrm{RR}=1.7,95 \% \mathrm{Cl} 1.2-$ 2.5). Conclusion: These results constitute an empirical validation of the concept of fast decliners in community-based $A D$ patients and justify the cutoff of 3 points for the definition of this condition.

Copyright $\odot 2007$ S. Karger AG, Basel
\end{abstract}

\section{Introduction}

A progressive cognitive decline is the main characteristic of Alzheimer's disease (AD). However, this decline widely varies among subjects at the onset of the disease and the distinction between slow and fast decliners has recently been emphasized [1]. In addition, the association between severe cognitive decline during the course of $\mathrm{AD}$ and a higher mortality rate has already been described [2-5].

On the whole, current findings suggest that fast decliners need particular clinical attention as they have a poorer prognosis with higher mortality rates [6]. Yet, the notion of fast decliners remains arbitrary and needs more validation. Indeed, such a category of patients was identified during post hoc analyses, in selected clinical samples participating in clinical trials [7]. These samples were composed of patients previously chosen because they presented specific characteristics and thus were not representative of the population. In addition, the previous studies on the relationship between cognitive decline and mortality have analyzed the decline over the whole course of the disease but not specifically at the phase following the diagnosis $[2,8,9]$ while this period of time seems the more relevant phase to provide adequate early information and to better manage the patient and his/her family.

\section{KARGER}

Fax +4161306 1234 E-Mail karger@karger.ch www.karger.com

\footnotetext{
Jean-François Dartigues

Inserm U593 - PAQUID - Case 11

146, rue Léo-Saignat, FR-33076 Bordeaux Cedex (France)

Tel. +33 5575715 02, Fax +33 557571486

E-Mail jean-francois.dartigues@isped.u-bordeaux2.fr
} 
Thus, the practical interest of the concept of fast decliner needs to be confirmed for its prognostic value at the time of $\mathrm{AD}$ diagnosis, in an unselected sample. Longitudinal population-based studies with long-term follow-ups are necessary to conduct such a validation. The French longitudinal PAQUID study [10] allowed us to analyze unselected new cases of AD occurring in the general population. Moreover, this cohort provides data on the evolution of cognitive performances over the 13 years of follow-up and thus the cognitive decline at the time of dementia diagnosis, as well as a long-term follow-up of the patients until death.

The aim of this study was to define a category of fast cognitive decliners at the time of $\mathrm{AD}$ diagnosis which best predicts mortality, in a sample of AD cases in the general population. We investigated different thresholds of decline to define when a subject could be considered as a fast decliner. To do so, we selected 245 subjects prospectively screened for AD from the PAQUID cohort and we focused on the relation between the annual rate of cognitive decline at the time of $\mathrm{AD}$ diagnosis and the risk of mortality.

\section{Methods}

\section{Study Design}

Our data came from the PAQUID (Personnes Agées QUID) cohort, a study on cerebral and functional aging. The detailed methodology of the PAQUID study has been described in full elsewhere [10]. The baseline visit began in 1988-1989.

Overall, 3,777 subjects accepted to participate and signed a written consent. At baseline, the participants were visited at home by a neuropsychologist and reinterviewed similarly 1 (in Gironde only), 3, 5, 8, 10 and 13 years after the baseline assessment.

An active research of dementia with confirmation by a senior neurologist was conducted at each follow-up as detailed below.

\section{Sample Selection}

In the present study, after exclusion of the 102 subjects demented at baseline, our sample included all the cases of possible or probable $\mathrm{AD}$ diagnosed between the 1-year visit and the 10year visit $(n=328)$. We did not include the demented subjects diagnosed after the 10 -year visit.

Let us refer to the visit of the diagnosis of AD as 'ADV'. In order to study the cognitive decline, subjects had to have been seen prior to ADV (ADV-1) and they had to have performed the global cognitive evaluation test [Mini Mental State Examination (MMSE)] at both visits. Finally, our sample included 245 subjects, after exclusion of 83 subjects with a missing value for MMSE either at ADV or at ADV-1. There was no statistical difference in age, sex or education between these 83 subjects and the rest of the sample.

\section{Data Collection and Dementia Diagnosis}

Interviews were performed at home by a trained psychologist with a standardized questionnaire, which contained sociodemographic characteristics, objective and subjective physical health, and a set of neuropsychological tests. This battery of tests included an evaluation of the global mental status (with the MMSE) [11], visual memory (Benton's Visual Retention Test) [12], visuospatial attention (Zazzo's Cancellation Test) [13], verbal fluency (Isaac Set Test) [14], and simple logical reasoning and attention (Wechsler's Digit Symbol Test) [15]. At the end of the neuropsychological interview, the psychologist used the DSM-IIIR checklist to select subjects suspected of being demented [16]. Those suspected cases were seen by a senior neurologist, blinded to the psychometric battery and functional assessment, to confirm or rule out the diagnosis of dementia. The specialist also specified the etiology of dementia using the NINCDS-ADRDA criteria for AD [17] and the Hachinski score for vascular dementia [18]. Cases were classified as probable or possible $\mathrm{AD}$, vascular dementia, and other types of dementia.

For all incident cases of dementia, the same procedure of diagnosis was used at each follow-up, but in order to increase the screening sensitivity, the criterion of a cognitive loss of 3 points in the MMSE score from the previous visit was added to qualify for neurological examination.

Finally, the survival status was collected throughout the follow-up for each participant up to the 13-year visit.

\section{Rate of Cognitive Decline: Calculation}

The annual rate of cognitive decline at the time of AD diagnosis was based on the MMSE score evolution between ADV-1 and ADV. This score ranges from 0 to 30 , and higher scores indicate better cognitive functioning. The annual rate of decline was calculated by subtracting the MMSE score obtained at ADV-1 from the score reached at ADV (in order to have a positive indicator, as the last measure is mostly lower than the previous one). Finally, the annual rate of cognitive decline was obtained by dividing this difference by the delay (in years) between the two visits.

Data Analysis

After describing the sample, we studied the association between the rate of cognitive decline and mortality. Survival time was considered from ADV to death for deceased subjects or to the 13 -year visit for those subjects not deceased. We tested 5 different thresholds of decline: a loss of at least 1,2, 3, 4 and 5 points per year in the MMSE score between ADV and the ADV-1.

Risk of mortality was assessed using Cox proportional hazards models [19]. For each of the 5 thresholds of cognitive decline investigated, we represented, on the same figure, estimated univariate survival curves and the curve corresponding to the loss of less than 1 point a year. Then, we controlled our models for potential confounding factors: age at ADV, sex, level of education (subjects were considered as highly educated if they had obtained the French primary school certificate, corresponding to about 7 years of schooling, which had previously been validated as the best cutoff for the prediction of dementia in the PAQUID study [20]), and MMSE score at ADV in two categories (less or more than 19). We previously examined whether age and MMSE scores should be modeled as continuous or categorical variables and we concluded that age should be used as a continuous variable and MMSE scores as a categorical variable with a cutoff of 19 . Validity of the propor-
Carcaillon/Pérès/Péré/Helmer/ Orgogozo/Dartigues 
tional hazard assumption was evaluated by testing interaction time-dependent variables and by a graphical evaluation.

Afterwards, we investigated if the category of fast decliners associated with a higher risk of mortality had other characteristics that could explain their faster decline. To do so, we compared slow and fast decliners regarding sex, age at inclusion and at ADV, education, MMSE score at ADV and ADV-1, social situation at ADV (family situation, type of residence, and number of close family members or friends), bed restriction at ADV, blindness and deafness at ADV, etiology of the disease (probable or possible $\mathrm{AD})$, symptomatic depression at ADV and ADV-1, medications at ADV and ADV-1 (antidepressants and anticholinergics).

\section{Results}

\section{Sample Description}

Our sample was composed of 245 subjects with AD. Table 1 presents the main sociodemographic and health characteristics of our sample. The mean age was 78.8 years at inclusion and 85.5 years at ADV. Seventy percent of the sample were women, $26.9 \%$ of the subjects were living alone, and $81.9 \%$ had more than 3 close family members or friends. Possible AD cases represented more than $52.0 \%$ of the total AD cases diagnosed. In all, 18.7\% suffered from symptomatic depression at ADV and 13.9\% at ADV-1.

\section{Variable of Interest}

The mean MMSE score was 17.5 at ADV and 23.2 at ADV-1 (table 1). The mean delay between these two visits was 2.2 years $(\mathrm{SD}=0.6)$ and ranged from 0.9 to 4.0 years. The annual loss of points in the MMSE score between ADV and ADV-1 was $2.2(\mathrm{SD}=2.3)$ on average and ranged from -3.3 (a gain of 3.3 points per year) to 16.1 points (fig. 1).

Regarding the different thresholds of decline, 205 subjects $(83.7 \%)$ lost 1 point or more per year in the MMSE score, 133 subjects (54.3\%) lost 2 points or more, 83 (33.9\%) lost 3 points or more, $44(18.0 \%)$ lost 4 points or more, and $21(8.6 \%)$ lost 5 points or more.

\section{Thresholds of Cognitive Decline and Risk of Mortality}

Between ADV and the 13-year visit, 167 (68.2 \%) subjects died. Considering the thresholds of a loss of 1, 2, 3, 4 and 5 points, 139 (67.8\%), 95 (71.4\%), 65 (78.3\%), 37 $(84.1 \%)$, and $17(81.0 \%)$ subjects, respectively, died in the category of fast decliners.

Table 2 presents both univariate and multivariate results of the Cox proportional hazards models for each of the 5 thresholds. The univariate relative risks (RRs) of death among subjects who lost at least more than 2, 3, 4 and 5 points were significantly increased. The risk of

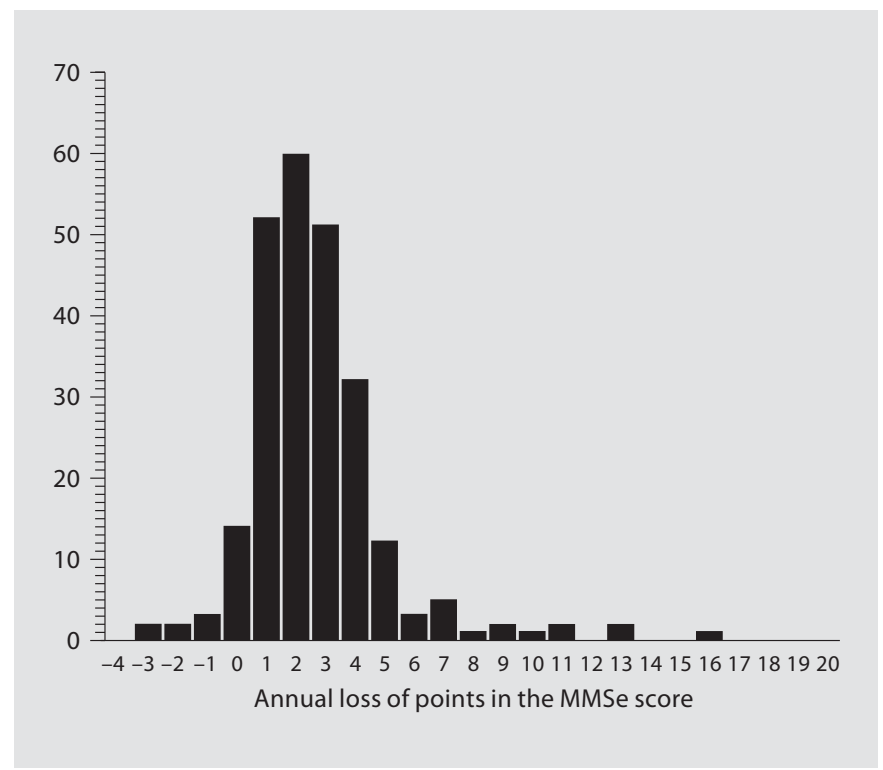

Fig. 1. Annual loss of points in the MMSE score prior to AD diagnosis.

Table 1. Sociodemographic and health characteristics of the subjects

\begin{tabular}{lrc}
\hline & $\mathrm{n}$ & $\%$ \\
\hline Female (vs. male) & 171 & 69.8 \\
Primary education level (vs. no diploma) & 122 & 49.8 \\
Family situation at ADV (alone vs. not alone) & 66 & 26.9 \\
Number of close family members or friends at & & \\
$\quad$ ADV (3 or more vs. less than 3) & 95 & 81.9 \\
Etiology (possible AD vs. probable AD) & 128 & 52.2 \\
Symptomatic depression at ADV & 34 & 18.7 \\
Symptomatic depression at ADV-1 & 32 & 13.9 \\
\hline & Mean & SD \\
\hline Age at baseline & 78.84 & 6.0 \\
Age at ADV & 85.48 & 6.0 \\
MMSE score at ADV & 17.48 & 5.5 \\
MMSE score at ADV-1 & 23.21 & 3.7 \\
\hline
\end{tabular}

death was not significantly different between slow and fast decliners with the threshold of 1 point. Figure 2 shows the estimated survival curves following ADV for the 5 thresholds of loss and for the loss of less than 1 point per year in the MMSE score. The survival time is different (shorter) for the decline of 3,4 and 5 points or more. 
Table 2. Univariate and multivariate risks of death for the 5 thresholds of cognitive decline

\begin{tabular}{|c|c|c|c|c|c|c|}
\hline & \multicolumn{3}{|c|}{ Univariate } & \multicolumn{3}{|c|}{ Multivariate } \\
\hline & $\mathrm{RR}$ & $95 \% \mathrm{CI}$ & $\mathrm{p}$ value & $\mathrm{RR}$ & $95 \% \mathrm{CI}$ & $\mathrm{p}$ value \\
\hline \multicolumn{7}{|l|}{ Model 1} \\
\hline Annual loss of points in the MMSE score $\geq 1$ & 1.19 & $0.79-1.79$ & 0.399 & 0.89 & $0.58-1.37$ & 0.593 \\
\hline Sex (male vs. female) & & & & 1.58 & $1.11-2.26$ & 0.012 \\
\hline Age at $\mathrm{ADV}$ & & & & 1.08 & $1.05-1.12$ & $<0.001$ \\
\hline At least primary education & & & & 1.23 & $0.90-1.69$ & 0.186 \\
\hline MMSE score at ADV $\geq 19$ & & & & 0.73 & $0.53-1.02$ & 0.063 \\
\hline \multicolumn{7}{|l|}{ Model 2} \\
\hline Annual loss of points in the MMSE score $\geq 2$ & 1.36 & $1.00-1.85$ & 0.048 & 1.24 & $0.90-1.71$ & 0.193 \\
\hline Sex (male vs. female) & & & & 1.59 & $1.11-2.27$ & 0.011 \\
\hline Age at $\mathrm{ADV}$ & & & & 1.09 & $1.05-1.12$ & $<0.001$ \\
\hline At least primary education & & & & 1.17 & $0.86-1.60$ & 0.326 \\
\hline MMSE score at ADV $\geq 19$ & & & & 0.79 & $0.57-1.10$ & 0.161 \\
\hline \multicolumn{7}{|l|}{ Model 3} \\
\hline Annual loss of points in the MMSE score $\geq 3$ & 1.78 & $1.30-2.43$ & $<0.001$ & 1.71 & $1.18-2.47$ & 0.005 \\
\hline Sex (male vs. female) & & & & 1.62 & $1.13-2.32$ & 0.009 \\
\hline Age at $\mathrm{ADV}$ & & & & 1.09 & $1.06-1.12$ & $<0.001$ \\
\hline At least primary education & & & & 1.06 & $0.77-1.47$ & 0.714 \\
\hline MMSE score at ADV $\geq 19$ & & & & 0.95 & $0.66-1.37$ & 0.779 \\
\hline \multicolumn{7}{|l|}{ Model 4} \\
\hline Annual loss of points in the MMSE score $\geq 4$ & 2.33 & $1.61-3.37$ & $<0.001$ & 2.11 & $1.39-3.20$ & $<0.001$ \\
\hline Sex (male vs. female) & & & & 1.57 & $1.10-2.24$ & 0.014 \\
\hline Age at $\mathrm{ADV}$ & & & & 1.08 & $1.05-1.12$ & $<0.001$ \\
\hline At least primary education & & & & 1.16 & $0.85-1.58$ & 0.363 \\
\hline MMSE score at ADV $\geq 19$ & & & & 0.93 & $0.65-1.33$ & 0.692 \\
\hline \multicolumn{7}{|l|}{ Model 5} \\
\hline Annual loss of points in the MMSE score $\geq 5$ & 3.11 & $1.95-5.21$ & $<0.001$ & 2.19 & $1.20-3.61$ & 0.009 \\
\hline Sex (male vs. female) & & & & 1.53 & $1.07-2.19$ & 0.019 \\
\hline Age at $\mathrm{ADV}$ & & & & 1.08 & $1.05-1.11$ & $<0.001$ \\
\hline At least primary education & & & & 1.21 & $0.89-1.65$ & 0.226 \\
\hline MMSE score at ADV $\geq 19$ & & & & 0.82 & $0.59-1.15$ & 0.258 \\
\hline
\end{tabular}

Controlled for age, sex, level of education, and MMSE score at ADV, the risk of death remained nonsignificant for the threshold of 1 point $(\mathrm{p}=0.593)$ and no longer reached significance for the threshold of 2 points $(\mathrm{p}=$ 0.193). For the thresholds of 3,4 and 5 points, the risk of death remained significantly increased after adjustments (respectively, RR $=1.7,95 \%$ CI $1.2-2.5 ; \mathrm{RR}=2.1,95 \% \mathrm{CI}$ 1.4-3.2; $\mathrm{RR}=2.1,95 \% \mathrm{CI} 1.2-3.6)$. As expected, the risk of death increased with age and was higher among men, whereas there was no association with MMSE score at $\mathrm{ADV}$, irrespective of the threshold.

\section{Characteristics of Fast Decliners}

We chose to define the category of fast decliners according to the 3-point threshold as it was the first threshold to significantly increase the risk of mortality. Table 3 presents sociodemographic and health characteristics of slow and fast decliners defined on the threshold of 3 points. Neither sex nor education was associated with the category of decline. However, fast decliners were older at ADV and at inclusion. Fast decliners had significantly lower MMSE scores at ADV (13.2 vs. 19.7) although they had similar scores at ADV-1 (23.5 vs. 23.0). In addition, fast decliners were more likely to be in a nursing home (44.6 vs. $14.8 \%$ ). No significant difference was observed between slow and fast decliners in terms of family situation and number of close family members or friends. Six subjects were bed restricted at ADV and they were all fast decliners. Yet, neither blindness nor deafness was related to the category of decline. More fast decliners than slow decliners (61.5 vs. $47.5 \%)$ were diagnosed as possible $\mathrm{AD}$ cases. Symptomatic depression at ADV and at ADV-1 was not associated with the category of decline. However, it is to be noted that $26.9 \%$ of the fast decliners suffered from 
Fig. 2. Kaplan-Meier survival curves after the diagnosis of AD stratified by the rate of cognitive decline.

Table 3. Characteristics of subjects using the threshold of 3 points

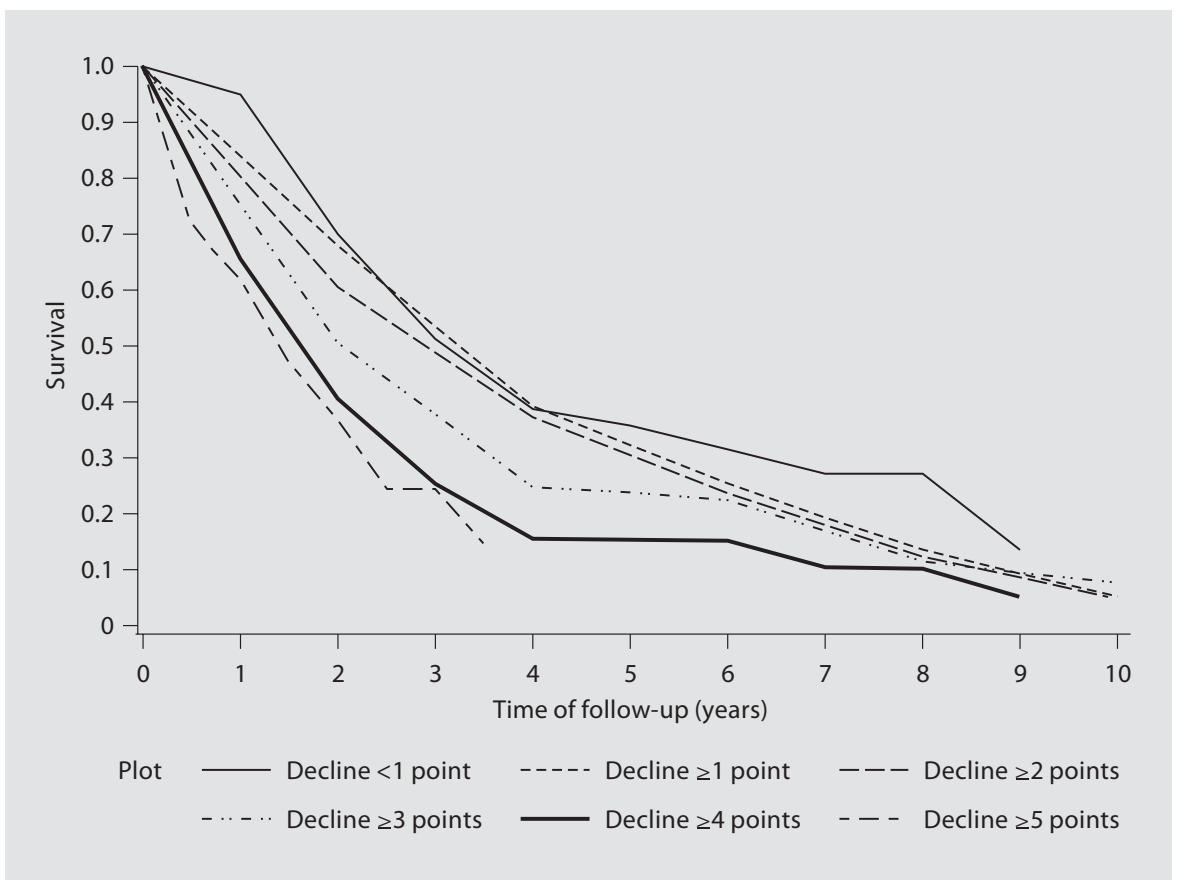

\begin{tabular}{|c|c|c|c|}
\hline & \multicolumn{2}{|l|}{ Threshold 3} & \multirow[t]{2}{*}{$\mathrm{p}$ value } \\
\hline & $<3(\mathrm{n}=162)$ & $\geq 3(\mathrm{n}=83)$ & \\
\hline Female (vs. male) & $111(68.5)$ & $60(72.3)$ & 0.543 \\
\hline Primary education level (vs. no diploma) & $75(46.3)$ & $47(56.6)$ & 0.126 \\
\hline Family situation at ADV (alone vs. not alone) & $48(29.6)$ & $18(21.7)$ & 0.185 \\
\hline Type of residence at ADV & & & $<0.001$ \\
\hline Home or old people's housing & $135(83.3)$ & $41(49.4)$ & \\
\hline Nursing home & $24(14.8)$ & $37(44.6)$ & \\
\hline Other & $3(1.9)$ & $5(6.0)$ & \\
\hline \multicolumn{4}{|l|}{ Close family members or friends at ADV } \\
\hline ( 3 or more vs. less than 3 ) & $66(84.6)$ & $29(76.3)$ & 0.276 \\
\hline Bed restricted at $\mathrm{ADV}$ & 0 & $6(7.4)$ & 0.001 \\
\hline Total blindness at ADV & $3(1.9)$ & $2(2.5)$ & 0.733 \\
\hline Deafness at ADV & $17(10.6)$ & $10(12.8)$ & 0.605 \\
\hline Etiology (possible AD vs. probable AD) & $77(47.5)$ & $51(61.5)$ & 0.039 \\
\hline Symptomatic depression at ADV & $20(15.4)$ & $14(26.9)$ & 0.071 \\
\hline Symptomatic depression at ADV-1 & $20(13.2)$ & $12(15.4)$ & 0.644 \\
\hline Hospitalized during the year before ADV & $51(31.7)$ & $43(54.4)$ & 0.001 \\
\hline Antidepressants at ADV & $31(20.0)$ & $15(19.7)$ & 0.963 \\
\hline Antidepressants at ADV-1 & $22(14.2)$ & $3(3.9)$ & 0.019 \\
\hline Anticholinergics at ADV & $27(17.4)$ & $22(28.9)$ & 0.044 \\
\hline Anticholinergics at ADV-1 & $28(18.1)$ & $17(22.4)$ & 0.438 \\
\hline Age at inclusion & $78.0 \pm 5.9$ & $80.5 \pm 6.0$ & 0.002 \\
\hline Age at ADV & $84.8 \pm 5.8$ & $86.8 \pm 6.1$ & 0.013 \\
\hline MMSE score at ADV & $19.7 \pm 3.5$ & $13.2 \pm 6.2$ & $<0.001$ \\
\hline MMSE score at ADV-1 & $23.0 \pm 3.8$ & $23.5 \pm 3.5$ & 0.323 \\
\hline
\end{tabular}

Figures in parentheses are percentages. 
symptomatic depression at ADV, compared with $15.4 \%$ of the slow decliners. Fast decliners had been more often hospitalized during the year before the diagnosis than slow decliners (54.4 vs. 31.7\%). Concerning medications, whereas slow and fast decliners were both treated with antidepressants at ADV, fast decliners were significantly less treated with antidepressants at ADV-1 (3.9vs. 14.2\%), and, compared with slow decliners, fast decliners were significantly more treated with anticholinergics at ADV (28.9 vs. $17.4 \%)$ although there was no difference at ADV-1.

\section{Discussion}

In this population-based prospective study, we found that in $\mathrm{AD}$ patients, fast cognitive decline at the time of the diagnosis was a strong prognostic factor for survival. The significant threshold of decline was a loss of 3 points or more per year in the MMSE score, while the thresholds of 2 or 1 points per year were not significantly associated with survival after appropriate adjustments. Taking into account the threshold of 3 points, we searched if specific characteristics could explain this faster decline. None of the sociodemographic characteristics appeared to be different using this threshold. Fast decliners were more likely to have been hospitalized during the year before ADV than slow decliners and were more often living in nursing homes. In addition, fast decliners were more often on anticholinergic drugs than slow decliners. This last result is consistent with the association, previously found in the PAQUID study, between anticholinergic drugs and low cognitive performances [21]. Moreover, as cholinergic drugs are assumed to avoid fast decline, this association between fast decline and anticholinergic drugs deserves further investigation. These findings constitute an empirical validation of the concept of fast decliners in community-based $\mathrm{AD}$ patients and justify the cutoff of 3 points for the definition of this condition.

The strength of this study is that it was conducted in an unselected sample of $\mathrm{AD}$ patients and that the decline of MMSE scores was effectively measured before the diagnosis of dementia and not only estimated as proposed by Doody et al. [22]. Some authors had already pointed out this 3-points-per-year threshold [7], but their data were not validated by a follow-up study.

The loss of 3 points or more in the MMSE score at the time of AD diagnosis may express an aggravation of the disease. One assumption for this is the faster progression of the underlying pathological process of AD. Indeed, the rate at which senile plaques and neurofibrillary tangles accumulate in the brain may increase and thus lead to a diminution of cognitive functions. Another assumption is the failure of compensatory mechanisms. It is well known that cognitive reserves decrease the deleterious effect of senile plaques on cognitive abilities [23, 24]. Thus, the collapse of these reserves could explain the decrease in cognitive functions.

Our study presents some limitations. It is known that before 1995, in many countries, subjects with Lewy body disease were often classified as cases of possible AD. In the PAQUID study, demented subjects with parkinsonism were excluded from the possible $\mathrm{AD}$ group. Therefore, only a minority of subjects with Lewy body disease may be included in the possible AD group. Hence, our results should not be affected by the presence of these subjects.

Moreover, we tested 5 different thresholds of decline; therefore, false-positive associations cannot be ruled out because of multiple testing. However, using the Bonferroni correction, the $\mathrm{p}$ value associated with the 3-point threshold remains significant as it has to be compared with 0.01 .

In the PAQUID study, subjects were visited every 2 or 3 years and thus, the actual date of diagnosis is imprecise. When a subject is diagnosed, he/she could have been demented for more than 2 years or only for a few months. Thus, one could be considered as a fast decliner and another could be considered as a slow decliner. This bias may lead to an overestimation of the relation between cognitive decline and mortality. However, our analyses were adjusted for cognitive level at ADV which prevents such an overestimation.

To conclude, the loss of 3 points per year in the MMSE score identifies a group of fast decliners with a higher risk of mortality. This category of patients consistently seems to have a more aggressive disease and thus needs particular attention at follow-up. In addition, their treatments must be adapted. As the great majority of our subjects were not treated with cholinesterase inhibitors, it would be interesting to know if fast decliners better respond to this type of medication [6]. Indeed, reducing the speed of cognitive decline may prevent the subjects from a precipitated death. Clinical trials should be conducted in order to determine whether or not the risk of death can be reduced in this category of fast decliners. 


\section{Acknowledgements}

The PAQUID project was funded by: ARMA (Bordeaux); Caisse Nationale d'Assurance Maladie des Travailleurs Salariés (CNAMTS); Conseil Général de la Dordogne; Conseil Général de la Gironde; Conseil Régional d'Aquitaine; Fondation de France; France Alzheimer (Paris); GIS Longévité; Institut National de la Santé et de la Recherche Médicale (INSERM); Mutuelle Générale de l'Education Nationale (MGEN); Mutualité Sociale Agricole (MSA); Novartis Pharma (France); SCOR Insurance (France).

\section{References}

1 Farlow MR, Hake A, Messina J, Hartman R, Veach J, Anand R: Response of patients with Alzheimer disease to rivastigmine treatment is predicted by the rate of disease progression. Arch Neurol 2001;58:417-422.

2 Schupf N, Tang MX, Albert SM, Costa R, Andrews H, Lee JH, Mayeux R: Decline in cognitive and functional skills increases mortality risk in nondemented elderly. Neurology 2005;65:1218-1226.

-3 Schupf N, Costa R, Tang MX, Andrews H, Tycko B, Lee JH, Mayeux R: Preservation of cognitive and functional ability as markers of longevity. Neurobiol Aging 2004;25:12311240.

4 Bassuk SS, Wypij D, Berkman LF: Cognitive impairment and mortality in the community-dwelling elderly. Am J Epidemiol 2000; 151:676-688.

5 Bruce ML, Hoff RA, Jacobs SC, Leaf PJ: The effects of cognitive impairment on 9-year mortality in a community sample. J Gerontol B Psychol Sci Soc Sci 1995;50:P289-P296.

-6 Farlow MR, Small GW, Quarg P, Krause A: Efficacy of rivastigmine in Alzheimer's disease patients with rapid disease progression: results of a meta-analysis. Dement Geriatr Cogn Disord 2005;20:192-197.

$\checkmark 7$ O'Hara R, Thompson JM, Kraemer HC, Fenn C, Taylor JL, Ross L, Yesavage JA, Bailey AM, Tinklenberg JR: Which Alzheimer patients are at risk for rapid cognitive decline? J Geriatr Psychiatry Neurol 2002;15: 233-238.

8 Wilson RS, Li Y, Aggarwal NT, McCann JJ, Gilley DW, Bienias JL, Barnes LL, Evans DA: Cognitive decline and survival in Alzheimer's disease. Int J Geriatr Psychiatry 2006;21: 356-362.
Larson EB, Shadlen MF, Wang L, McCormick WC, Bowen JD, Teri L, Kukull WA: Survival after initial diagnosis of Alzheimer disease. Ann Intern Med 2004;140:501-509.

10 Dartigues JF, Gagnon M, Michel P, Letenneur L, Commenges D, Barberger-Gateau P, Auriacombe S, Rigal B, Bedry R, Alperovitch $A$, et al: The Paquid research program on the epidemiology of dementia. Methods and initial results. Rev Neurol (Paris) 1991;147:225230.

11 Folstein MF, Folstein SE, McHugh PR: 'Minimental state'. A practical method for grading the cognitive state of patients for the clinician. J Psychiatr Res 1975;12:189-198.

12 Benton A: Manuel pour l'application du test de rétention visuelle. Applications cliniques et expérimentales, ed 2. Paris, Centre de Psychologie Appliquée, 1965.

13 Zazzo R: Test des deux barrages. Actualités pédagogiques et psychologiques. Neuchâtel, Delachaux et Niestle, 1974, vol 7.

14 Isaacs B, Kennie AT: The Set test as an aid to the detection of dementia in old people. $\mathrm{Br} \mathrm{J}$ Psychiatry 1973;123:467-470.

15 Wechsler D: WAIS-R Manual. New York, Psychological Corporation, 1981.

16 Pichot P: DSM-III: the 3rd edition of the Diagnostic and Statistical Manual of Mental Disorders from the American Psychiatric Association. Rev Neurol (Paris) 1986;142: 489-499.
17 McKhann G, Drachman D, Folstein M, Katzman R, Price D, Stadlan EM: Clinical diagnosis of Alzheimer's disease: report of the NINCDS-ADRDA Work Group under the auspices of Department of Health and Human Services Task Force on Alzheimer's Disease. Neurology 1984;34:939-944.

18 Hachinski VC, Iliff LD, Zilhka E, Du Boulay GH, McAllister VL, Marshall J, Russell RW, Symon L: Cerebral blood flow in dementia. Arch Neurol 1975;32:632-637.

19 Cox D: Regression models in life tables (with discussion). J R Stat Soc 1972;34:187-220.

- 20 Letenneur L, Gilleron V, Commenges D, Helmer C, Orgogozo M, Dartigues JF: Are sex and educational level independent predictors of dementia and Alzheimer's disease? Incidence data from the PAQUID project. J Neurol Neurosurg Psychiatry 1999;66:177183.

21 Lechevallier-Michel N, Molimard M, Dartigues JF, Fabrigoule C, Fourrier-Reglat A: Drugs with anticholinergic properties and cognitive performance in the elderly: results from the PAQUID Study. Br J Clin Pharmacol 2005;59:143-151.

22 Doody RS, Massman P, Dunn JK: A method for estimating progression rates in Alzheimer disease. Arch Neurol 2001;58:449-454.

23 Stern Y, Tang MX, Denaro J, Mayeux R: Increased risk of mortality in Alzheimer's disease patients with more advanced educational and occupational attainment. Ann Neurol 1995;37:590-595.

24 Stern Y, Gurland B, Tatemichi TK, Tang MX, Wilder D, Mayeux R: Influence of education and occupation on the incidence of Alzheimer's disease. JAMA 1994;271:1004-1010. 